\title{
LAS ELECCIONES MUNICIPALES Y AUTONOMICAS DE 1983
}

CUAdRo NúM. 1

RESULTADO DE LAS ELECCIONES MUNICIPALES Y AUTONOMICAS 1983

(En porcentaje sobre voto válido)

Autonómicas 1983

Censo: 14.910 .850

Voto válido: $10.200 .877(68,4)$ *

Blancos y nulos: $179.415(1,2)$ *

Abstención: $4.530 .558(30,4)$

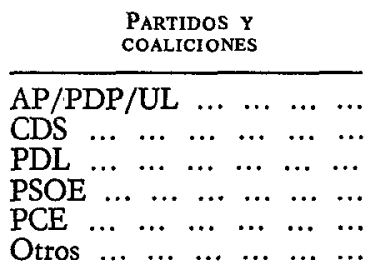

\begin{tabular}{c}
$\begin{array}{c}\text { Municipales } \\
1983\end{array}$ \\
\hline 25,8 \\
1,8 \\
0,8 \\
43,2 \\
8,2 \\
20,1
\end{tabular}

\begin{tabular}{rrr}
$\begin{array}{c}\text { Autonomicas } \\
1983\end{array}$ & & \multicolumn{1}{c}{$\begin{array}{c}\text { Generales } \\
1982\end{array}$} \\
\cline { 1 - 1 } 33,7 & & 31,5 \\
3,2 & & 3,7 \\
1,4 & & UCD 7,9 \\
48,1 & & 49,1 \\
4,7 & & 3,8 \\
8,9 & & 4,0 \\
\cline { 1 - 1 } 100,0 & & 100,0
\end{tabular}

* Porcentaje sobre el censo.

FuENTE: Cuadros elaborados en base a datos del Ministerio del Interior 


\section{LAS ELECCIONES AUTONOMICAS POR PROVINCIAS}

Cuadro núM. 2

\section{Asturias}

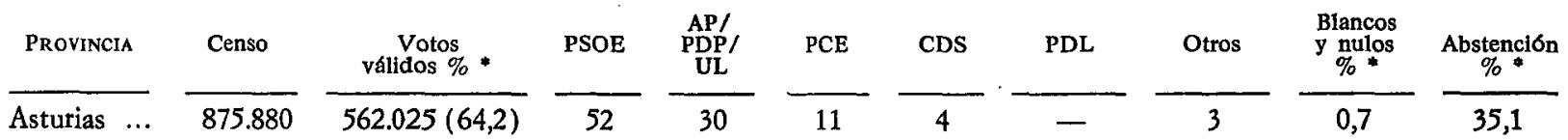

CuAdro NúM. 3

Aragón

\begin{tabular}{|c|c|c|c|c|c|c|c|c|c|c|c|}
\hline Provincia & Censo & $\begin{array}{l}\text { Votos } \\
\text { vallidos \% * }\end{array}$ & PSOE & $\begin{array}{c}\text { AP }{ }_{\text {PDP }} \\
\text { UL }\end{array}$ & PAR & PCE & CDS & PDL & Otros & $\begin{array}{l}\text { Blancos } \\
\text { y nulos } \\
\%_{*}^{*}\end{array}$ & $\underset{\%}{\operatorname{Abstencion}}$ \\
\hline $\begin{array}{l}\text { Huesca ... } \\
\text { Teruel .... } \\
\text { Zaragoza ... }\end{array}$ & $\begin{array}{l}170.940 \\
123.777 \\
625.753\end{array}$ & $\begin{array}{r}116.007(67,8) \\
81.443(65,8) \\
407.280(65,1)\end{array}$ & $\begin{array}{l}49 \\
39 \\
48\end{array}$ & $\begin{array}{l}27 \\
31 \\
20\end{array}$ & $\begin{array}{l}13 \\
23 \\
22\end{array}$ & $\begin{array}{l}5 \\
2 \\
4\end{array}$ & $\begin{array}{l}4 \\
4 \\
3\end{array}$ & $\frac{1}{-}$ & $\begin{array}{l}1 \\
1 \\
3\end{array}$ & $\begin{array}{l}1,6 \\
1,6 \\
1,3\end{array}$ & $\begin{array}{l}30,5 \\
32,6 \\
33,6\end{array}$ \\
\hline
\end{tabular}

CuAdro núm. 4

\section{BaLeares}

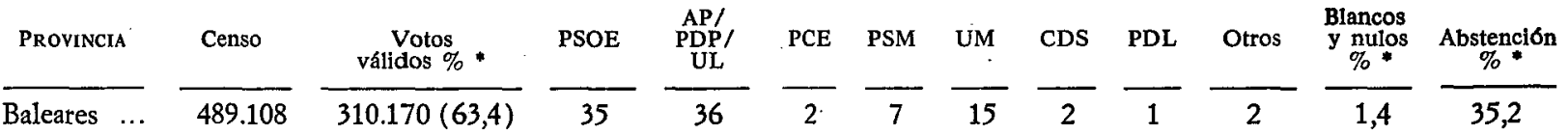

CuAdro NúM. 5

\section{Canarias}

\begin{tabular}{|c|c|c|c|c|c|c|c|c|c|c|}
\hline Provincia & Censo & $\begin{array}{l}\text { Votos } \\
\text { váliđos \% * }\end{array}$ & PSOE & $\begin{array}{c}\text { AP/ } \\
\text { PDP } \\
\text { UL }\end{array}$ & PCE & CDS & UPC & Otros & $\begin{array}{l}\text { Blancos } \\
\text { y nulos } \\
\%^{*}\end{array}$ & $\underset{\%}{\text { Abstención }}$ \\
\hline $\begin{array}{l}\text { Las Palmas. } \\
\text { Tenerife ... }\end{array}$ & $\begin{array}{l}477.326 \\
463.215\end{array}$ & $\begin{array}{l}291.413(00,0) \\
276.874(60,0)\end{array}$ & $\begin{array}{l}39 \\
44\end{array}$ & $\begin{array}{l}27 \\
31\end{array}$ & $\begin{array}{l}4 \\
4\end{array}$ & $\begin{array}{l}6 \\
8\end{array}$ & $\begin{array}{l}9 \\
8\end{array}$ & $\begin{array}{r}15 \\
4\end{array}$ & $\begin{array}{l}1,7 \\
1,3\end{array}$ & $\begin{array}{l}37,2 \\
39,0\end{array}$ \\
\hline
\end{tabular}




\section{Cuadro núm. 6}

\begin{tabular}{|c|c|c|}
\hline Provincia & Censo & $\begin{array}{l}\text { Votos } \\
\text { válidos } \% \text { * }\end{array}$ \\
\hline $\begin{array}{l}\text { Albacete ... } \\
\text { C. Real ... } \\
\text { Cuenca .... } \\
\text { Guadalajara. } \\
\text { Toledo ..... }\end{array}$ & $\begin{array}{l}248.239 \\
353.190 \\
165.095 \\
110.325 \\
353.002\end{array}$ & $\begin{array}{r}172.734(69,6) \\
245.873(68,3) \\
121.763(73,7) \\
80.209(72,7) \\
269.493(76,3)\end{array}$ \\
\hline
\end{tabular}

Cuadro núm. 7

\begin{tabular}{|c|c|c|}
\hline Provincia & Censo & $\begin{array}{l}\text { Votos } \\
\text { válidos } \% *\end{array}$ \\
\hline Avila & 145.240 & $103.759(71,4)$ \\
\hline Burgos & 279.730 & $177.984(63,6)$ \\
\hline León .. & 409.425 & $258.254(63,1)$ \\
\hline Palencia ... & 144.773 & $104.016(71,9)$ \\
\hline Salamanca... & 208.077 & $200.291(71,5)$ \\
\hline Segovia & 115.122 & $83.635(72,7)$ \\
\hline Sori & 80.48 & $52.513(65,3)$ \\
\hline Valladolid. & 361.30 & $249.223(68,9)$ \\
\hline Zamora .. & 183.051 & $124.959(68,3)$ \\
\hline
\end{tabular}

\section{Cuadro núm. 8}

\begin{tabular}{|c|c|c|c|c|c|c|c|c|c|c|c|}
\hline Provincia & Censo & válidos $\% *$ & PSOE & $\begin{array}{l}\mathrm{AP} / \\
\mathrm{PDP}^{\prime \prime}\end{array}$ & $\mathrm{EU}$ & PCE & CDS & PDL & Otros & $\begin{array}{l}\text { Biancos } \\
\text { y nulos } \\
. \%\end{array}$ & $\underset{\%}{\text { Abstención }}$ \\
\hline Cáceres .... & $\begin{array}{l}311.878 \\
469128\end{array}$ & $\begin{array}{l}220.429(70,7) \\
336904(718)\end{array}$ & $\begin{array}{l}48 \\
57\end{array}$ & 29 & 16 & $\begin{array}{l}4 \\
8\end{array}$ & 2 & $=$ & 1 & $\begin{array}{l}1,0 \\
10\end{array}$ & $\begin{array}{l}28,3 \\
272\end{array}$ \\
\hline
\end{tabular}

\section{Castilla-La Mancha}

\begin{tabular}{|c|c|c|c|c|c|c|}
\hline $\begin{array}{l}\text { AP' } \\
\text { PDP } \\
\text { UL }\end{array}$ & PCE & CDS & PDL & Otros & $\begin{array}{l}\text { Blancos } \\
\text { y nulos } \\
\%_{\%}^{*}\end{array}$ & $\begin{array}{c}\text { Abstención } \\
\%_{\%}^{*}\end{array}$ \\
\hline 37 & 7 & 2 & - & - & 1,3 & 29,1 \\
\hline 39 & 6 & 4 & - & - & 1,3 & 30,4 \\
\hline 44 & 6 & 2 & - & 6 & 1,5 & 24,7 \\
\hline 47 & 7 & 4 & 2 & - & 1,8 & 25,5 \\
\hline 42 & 8 & 3 & 3 & - & 1,2 & 22,5 \\
\hline
\end{tabular}

\section{Castilia-León}

AP/

\begin{tabular}{c} 
PDP/ \\
UL \\
\hline 42 \\
46 \\
36 \\
47 \\
38 \\
47 \\
44 \\
35 \\
41
\end{tabular}

PDL
$\frac{8}{8}$
$\frac{5}{5}$
$\frac{5}{6}$

\begin{tabular}{c} 
PCE \\
\hline 3 \\
\hline 3 \\
3 \\
5 \\
2 \\
2 \\
2 \\
5 \\
2
\end{tabular}

\begin{tabular}{r} 
CDS \\
\hline 24 \\
5 \\
2 \\
5 \\
4 \\
6 \\
8 \\
5 \\
8
\end{tabular}

\begin{tabular}{c} 
Otros \\
\hline$\frac{1}{6}$ \\
2 \\
$\frac{6}{6}$ \\
2 \\
2
\end{tabular}

Blancos

$y$ nulos

\begin{tabular}{|c|c|}
\hline$\%{ }^{*}$ & $\%$ \\
\hline 1,4 & 27,1 \\
\hline 1,9 & 34,5 \\
\hline 1,6 & 35,3 \\
\hline 1,5 & 26,6 \\
\hline 1,5 & 27,0 \\
\hline 2,4 & 24,9 \\
\hline 2,2 & 32,5 \\
\hline 1,6 & 29,4 \\
\hline 1,7 & 30,0 \\
\hline
\end{tabular}

\section{ExTREMADURA}


Cuadro núm. 9

MAdRID

$\frac{\text { Provincia }}{\text { Madrid … }} \frac{\text { Censo }}{3.381 .610} \frac{\begin{array}{c}\text { Votos } \\ \text { vallidos \% }\end{array}}{2.326 .390(68,8)} \frac{\text { PSOE }}{51} \frac{\begin{array}{c}\text { AP/ } \\ \text { PDP/ } \\ \text { UL }\end{array}}{34} \frac{\text { PCE }}{9} \frac{\text { CDS }}{3} \quad \frac{\text { PDL }}{2} \frac{\text { Otros }}{1} \frac{\begin{array}{c}\text { Blancos } \\ \text { y nulos } \\ \% *\end{array}}{1,0} \frac{\begin{array}{c}\text { Abstención } \\ \% *\end{array}}{30,3}$

CuAdro núm. 10

MurCia

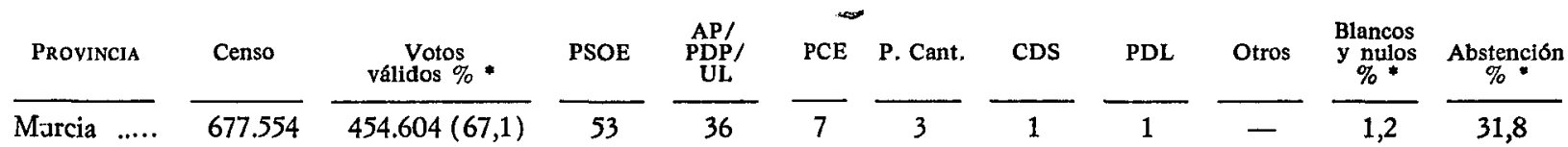

CuAdro NúM. 11

Navarra

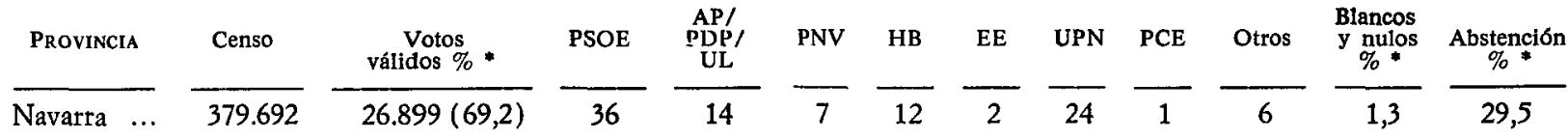

Cuadro núm. 12

La Rioja

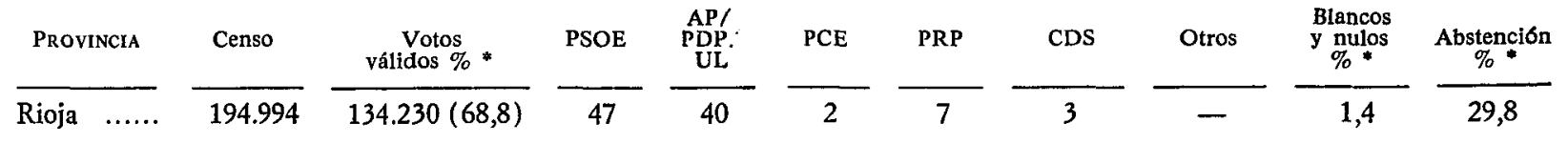




\section{Cuadro núm. 13}

\section{VALENCIANA}

\begin{tabular}{|c|c|c|c|c|c|c|c|c|c|c|c|}
\hline Provincia & Censo & $\begin{array}{c}\text { Votos } \\
\text { válidos \% * }\end{array}$ & PSOE & $\begin{array}{l}\text { AP/ } \\
\text { PDP/ } \\
\text { UL }\end{array}$ & UPV & PCE & CDS & PDL & Otros & $\begin{array}{c}\text { Blancos } \\
\text { y nulos } \\
\%_{*}^{*}\end{array}$ & $\underset{\%^{*}}{\text { Abstención }}$ \\
\hline $\begin{array}{ll}\text { Alicante } & . . . \\
\text { Castellón } & . . . \\
\text { Valencia } & . . .\end{array}$ & $\begin{array}{r}817.967 \\
322.614 \\
1.515 .370\end{array}$ & $\begin{array}{r}569.353(69,6) \\
237.146(73,5) \\
1.094 .925(72,3)\end{array}$ & $\begin{array}{l}55 \\
49 \\
51\end{array}$ & $\begin{array}{l}31 \\
34 \\
32\end{array}$ & $\begin{array}{l}2 \\
5 \\
3\end{array}$ & $\begin{array}{l}7 \\
6 \\
8\end{array}$ & $\begin{array}{l}2 \\
2 \\
2\end{array}$ & $\begin{array}{l}2 \\
4 \\
1\end{array}$ & $\frac{1}{3}$ & $\begin{array}{l}1,0 * \\
1,4 \\
1,1\end{array}$ & $\frac{29,4}{26,6}$ \\
\hline
\end{tabular}

Cuadro núm. 14

\section{Cantabria}

\begin{tabular}{|c|c|c|c|c|c|c|c|c|c|c|c|}
\hline Provincia & Censo & $\begin{array}{l}\text { Votos } \\
\text { validos \% }\end{array}$ & PSOE & $\begin{array}{l}\text { AP' } \\
\text { PDP } \\
\text { UL }\end{array}$ & PRC & PCE & $\cos$ & PDL & Otros & $\begin{array}{c}\text { Blancos } \\
y_{\%^{*}}\end{array}$ & $\underset{\%^{\star}}{\text { Abstención }}$ \\
\hline Cantabria... & 384.993 & $278.777(72,4)$ & 39 & 44 & 7 & 4 & 2 & 2 & 2 & 1,5 & 26,1 \\
\hline
\end{tabular}

Porcentaje sobre censo.
FUENTE: No se han publicado todavia cifras officiales de estas elecciones. Los cuadros de datos están elaborados en base a datos proporcionados por el Ministerio del Interior. 


\section{PRINCIPALES PARTIDOS Y COALICIONES}

PRESENTES EN ESTAS ELECCIONES

A PARLAMENTOS AUTONOMOS DE 1983

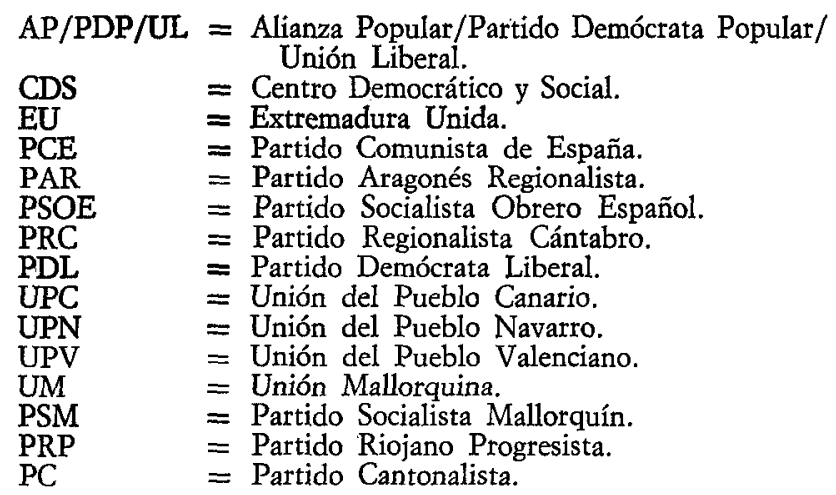

Fernando Ollero Butler 